\title{
Decomposition of complete bipartite graphs into open trails
}

\author{
Sylwia Cichacz; Agnieszka Görlich \\ AGH University of Science and Technology, Al. Mickiewicza 30, 30-059 Kraków, Poland
}

January 23, 2008

\begin{abstract}
It has been showed in [4] that any bipartite graph $K_{a, b}$, where $a, b$ are even is decomposable into closed trails of prescribed even lengths. In this article we consider the corresponding question for open trails. We prove a necessary and sufficient condition for graphs $K_{a, b}$ to be decomposable into edge-disjoint open trails of positive lengths (less than $a b$ ) whenever these lengths sum up to the size of the graph $K_{a, b}$. Let $K_{a, a}^{\prime}:=K_{a, a}-I_{a}$ for any 1-factor $I_{a}$. We also prove that $K_{a, a}^{\prime}$ for odd $a$ can be decomposed in a similar manner.
\end{abstract}

\section{Introduction}

Consider a simple graph $G$ whose size we denote by $e(G)$. Write $V(G)$ for the vertex set and $E(G)$ for the edge set of a graph $G$.

We say that a graph $G$ is Eulerian if there exists a closed trail through every edge of $G$. Here and subsequently, a trail $T$ of length $n$ we identify with a sequence $\left(v_{1}, v_{2}, \ldots, v_{n+1}\right)$ of vertices of $T$ such that $v_{i} v_{i+1}$ are distinct edges of $T$ for $i=1,2, \ldots, n$. Notice that we do not require the $v_{i}$ to be distinct. A trail $T$ is closed if $v_{1}=v_{n+1}$ and $T$ is open if $v_{1} \neq v_{n+1}$.

However, closed trail will be regarded as an Eulerian graph of size $n$. A graph $G$ is said to be even if the degrees of all its vertices are even. By

*Corresponding author. E-mail: cichacz@agh.edu.pl 
Euler's theorem, a connected even graph is Eulerian (i.e. contains a closed trail passing through all its edges exactly once).

A sequence of positive integers $\tau=\left(t_{1}, t_{2}, \ldots, t_{p}\right)$ is called admissible for a graph $G$ if it adds up to $e(G)$ and for each $i \in\{1, \ldots, p\}$ there exists an open trail of length $t_{i}$ in $G$. Let $\tau=\left(t_{1}, t_{2}, \ldots, t_{p}\right)$ be an admissible sequence for $G$. If $G$ is edge-disjointly decomposable into open trails $T_{1}, T_{2}, \ldots, T_{p}$ of lengths $t_{1}, t_{2}, \ldots, t_{p}$ respectively, then $\tau$ is called realizable in $G$ and the sequence $\left(T_{1}, T_{2}, \ldots, T_{p}\right)$ is said to be $a G$-realization of $\tau$ or a realization of $\tau$ in $G$.

Let $K_{a, b}$ be the complete bipartite graph with two sets of vertices $A$ and $B$ such that $|A|=a$ and $|B|=b$. In our paper we prove a necessary and sufficient condition for graphs $K_{a, b}$ to be decomposable into edge-disjoint open trails of positive lengths $t_{1}, t_{2}, \ldots t_{p}$ for any admissible sequence $\tau=$ $\left(t_{1}, t_{2}, \ldots t_{p}\right)$.

Such problems were first investigated by P.N. Balister.

Theorem 1 ([1]) Let $L=\sum_{i=1}^{p} t_{i}, t_{i} \geqslant 3$, with $L=\left(\begin{array}{l}n \\ 2\end{array}\right)$ when $n$ is odd and $\left(\begin{array}{l}n \\ 2\end{array}\right)-\frac{n}{2}-2 \leqslant L \leqslant\left(\begin{array}{l}n \\ 2\end{array}\right)-\frac{n}{2}$ when $n$ is even. Then we can write some subgraph of $K_{n}$ as an edge union of circuits of lengths $t_{1}, \ldots, t_{p}$.

Theorem 2 ([2]) The following conditions are both necessary and sufficient for packing $\bigcup_{i=1}^{p} P_{l_{i}}$ into $K_{n}$ with endpoints mapped to distinct vertices:

$L=\left(\begin{array}{l}n \\ 2\end{array}\right)$ or $L \leqslant\left(\begin{array}{l}n \\ 2\end{array}\right)-3$ if $r=0$,

$L \leqslant\left(\begin{array}{c}n \\ 2\end{array}\right)-\frac{n}{2}$ if $r>0$ and $r$ (or $n$ ) is even,

$L \leqslant\left(\begin{array}{l}n \\ 2\end{array}\right)-p$ if $r$ (or $n$ ) is odd:

where $n=2 p+r$ and $L=\sum_{i=1}^{p} l_{i}$. In particular, $L \leqslant\left(\begin{array}{c}n-1 \\ 2\end{array}\right)$ is always sufficient.

A motivation and applications of Theorems 1 and 2 can be found in problems concerning vertex-distinguishing proper edge-coloring of graphs.

A similar theorem for the closed trails has been proved in [4] by M. Horňák and M. Woźniak.

Theorem 3 ([4]) If $a, b$ are positive even integers, then if $\sum_{i=1}^{p} t_{i}=a b$ and there is a closed trail of length $t_{i}$ in $K_{a, b}$ (for all $i \in\{1, \ldots, p\}$ ), then $K_{a, b}$ can be (edge-disjointly) decomposed into closed trails $T_{1}, T_{2}, \ldots, T_{p}$ of lengths $t_{1}, t_{2}, \ldots, t_{p}$ respectively.

This problem is also solved by S. Cichacz for directed bipartite graphs and bipartite multigraphs, see [3]. 
Let $K_{a, a}$ be a complete bipartite graph and let $I_{a}$ denote a 1-factor in $K_{a, a}$. We denote by $K_{a, a}^{\prime}$ a graph $K_{a, a}-I_{a}$.

\section{Decomposition of bipartite graphs into open trails}

There is no loss of generality in assuming that $a \leq b$.

Let us observe that in any complete bipartite graph $K_{a, b}$ different from $K_{1,1}$ and $K_{2, b}$ for odd $b$ does not exist an open trail of length $a b$. Hence, $p \geq 2$ for each admissible sequence $\tau=\left(t_{1}, \ldots, t_{p}\right)$ for each graph $K_{a, b}$ different from $K_{1,1}$ and $K_{2, b}$ for any odd $b$.

Theorem 4 For each complete bipartite graph $K_{a, b}$ and for each admissible sequence $\tau=\left(t_{1}, \ldots, t_{p}\right)$ for $K_{a, b}$ there exists a realization of $\tau$ in $K_{a, b}$ if and only if one of the following conditions holds:

$1^{0} a=1$ or

$2^{0} a$ and $b$ are both even.

Let $A:=\left\{x_{1}, \ldots, x_{a}\right\}$ and $B:=\left\{v_{1}, \ldots, v_{b}\right\}$.

Necessity. We show that if $a>1$ and $a$ or $b$ is odd then there exists an admissible sequence $\tau$ for $K_{a, b}$ such that there is no realization for $\tau$ in $K_{a, b}$. We divide this proof into several parts:

A. Let us assume that $a=2$ and $b$ is odd. It can be easily seen that there exists an open trail of length two in $K_{2, b}$ and because of Euler's theorem there exists an open trail of length $(2 b-2)$ in $K_{2, b}$. Hence $\tau:=(2,2 b-2)$ is an admissible sequence for $K_{2, b}$ but $\tau$ is not realizable in $K_{2, b}$.

B. Let $a \geq 3$ and $b \geq 3$. Assume first that $a$ is odd while $b$ is even. Thus, $d\left(x_{i}\right)$ is even for any $i \in\{1, \ldots, a\}$ and $d\left(v_{j}\right)$ is odd for any $j \in\{1, \ldots, b\}$. Let $G_{1}$ be a subgraph of $K_{a, b}$ induced by the set of vertices $\left\{x_{1}, v_{1}, v_{2}, \ldots, v_{b-1}\right\}$ (see fig. 1). Let $G^{\prime}:=K_{a, b}-E\left(G_{1}\right)$. Observe that the only two vertices in $G^{\prime}$ of odd degree are $x_{1}$ and $v_{b}$. Thus, in $K_{a, b}$ there exists an open trail of length $(a b-b+1)$. Moreover, there exists an open trail of length $(b-1)$, but a sequence $\tau:=(b-1, a b-b+1)$ is not realizable in $K_{a, b}$ (because if $T_{1}$ denotes an open trail of length $(b-1)$ in $K_{a, b}$, then in $K_{a, b}-E\left(T_{1}\right)$ there are at least 

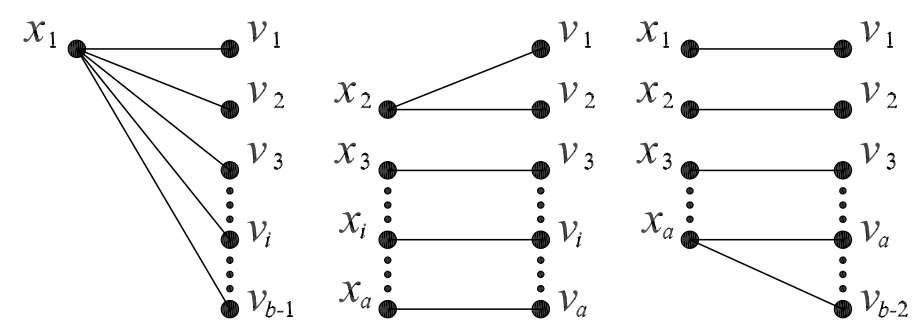

Figure 1: Subgraphs $G_{1}, G_{2}$ and $G_{3}$.

four vertices of odd degree). Analogously we show that such sequence $\tau$ is not realizable in $K_{a, b}$ for $a$ even and $b$ odd.

C. Let $a \geq 3$ and $b \geq 3$ be both odd. Let us consider two subcases:

a) $a=b$. Let $G_{2}$ be a subgraph of $K_{a, a}$ with the vertex set $V\left(G_{2}\right)=$ $\left\{x_{2}, \ldots, x_{a}, v_{1}, \ldots, v_{a}\right\}$ and the edge set $E\left(G_{2}\right)=\left\{x_{2} v_{1}, x_{2} v_{2}, x_{3} v_{3}, \ldots, x_{i} v_{i}\right.$, $\left.\ldots, x_{a} v_{a}\right\}$ (see fig. 1). In $K_{a, a}-E\left(G_{2}\right)$ there exist only two vertices of odd degree, namely $x_{1}$ and $x_{2}$. Hence in $K_{a, a}$ is an open trail of length $\left(a^{2}-a\right)$. There is also an open trail of length $a$ in $K_{a, a}$. But the sequence $\tau:=\left(a, a^{2}-a\right)$ is not realizable in $K_{a, a}$.

b) $a<b$. Let $G_{3}$ be a subgraph of $K_{a, b}$ with $V\left(G_{3}\right)=\left\{x_{1}, \ldots, x_{a}, v_{1}, \ldots, v_{b-2}\right\}$ and with $E\left(G_{3}\right)=\left\{x_{1} v_{1}, x_{2} v_{2}, x_{3} v_{3}, \ldots, x_{a} v_{a}, x_{a} v_{a+1}, \ldots, x_{a} v_{b-3}, x_{a} v_{b-2}\right\}$. Observe that $d_{G_{3}}\left(x_{1}\right)=\ldots=d_{G_{3}}\left(x_{a-1}\right)=d_{G_{3}}\left(v_{1}\right)=\ldots=d_{G_{3}}\left(v_{b-2}\right)=1$ and $d_{G_{3}}\left(x_{a}\right)=b-a-1$ (see fig. 1). Hence, in $K_{a, b}-E\left(G_{3}\right)$ the only two vertices of odd degree are $v_{b-1}$ and $v_{b}$. Notice that we allow $a=b-2$. This implies that there exists an open trail of length $(a b-b+2)$ in $K_{a, b}$. Obviously, in $K_{a, b}$ exists an open trail of length $(b-2)$. However, an edge-disjoint decomposition of $K_{a, b}$ into open trails of lengths $(b-2)$ and $(a b-b+2)$ does not exist.

Sufficiency. Assume first that $a=1$. It can be easily seen that $K_{1, b}$ is arbitrarily decomposable into open trails of length one and two.

From now on, let us assume that $G$ is any complete bipartite graph $K_{a, b}$ such that $a$ and $b$ are even. Let $\tau=\left(t_{1}, \ldots, t_{p}\right)$ be a sequence of positive integers such that $\sum_{i=1}^{p} t_{i}=a b$ and $p \geq 2$. We show that there exists a $\tau$-realization in $K_{a, b}$. We consider the following cases:

A. Let us suppose that $t_{i}$ is even for any $i \in\{1, \ldots, p\}$.

Case $I$. Assume now that $t_{i}$ is not an even multiplicity of $b$ for any $i \in$ 
$\{1, \ldots, p\}$. Consider a sequence

$$
\begin{gathered}
V:=\left(v_{1}, x_{1}, v_{2}, x_{2}, v_{3}, x_{1}, \ldots, x_{1}, v_{b}, x_{2},\right. \\
\left.v_{1}, x_{3}, v_{2}, x_{4}, v_{3}, x_{3}, \ldots, x_{3} v_{b}, x_{4}, \ldots, v_{1}, x_{a-1}, \ldots, x_{a-1}, v_{b}, x_{a}, v_{1}\right) .
\end{gathered}
$$

Clearly, this sequence of vertices creates an Eulerian trail in $K_{a, b}$. We show
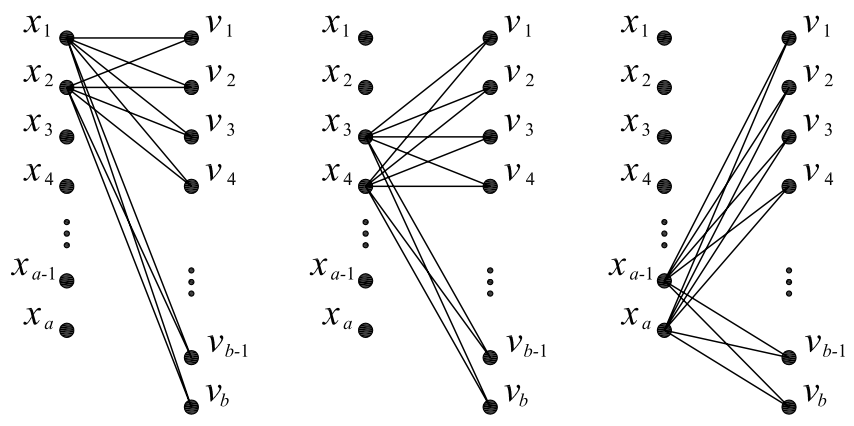

Figure 2: Sequence $V$.

that we can part $V$ into subsequences $V_{1}, \ldots, V_{p}$ such that for any $i \in\{1, \ldots, p\}$ the set of vertices in $V_{i}$ describes an open trail $T_{i}$ in $K_{a, b}$ of length $t_{i}$ and $T_{1}, \ldots, T_{p}$ are edge-disjoint subgraphs of $K_{a, b}$ (see fig 2 ).

Let us start at the following observation: let $W=\left(w_{1}, \ldots, w_{k}\right) \subset V$ be a subsequence of consecutive elements of $V$ such that $w_{1}=w_{k}=v_{i}$ for some $i \in\{1, \ldots, b\}$. The set of vertices in $W$ creates a closed trail in $K_{a, b}$ of length $m \cdot b$ for some even $m \leq a$.

We will define subsequences $V_{1}, \ldots, V_{p}$ of $V$. Let $V_{1}$ contain $\left(t_{1}+1\right)$ first elements of $V$ so it starts at $v_{1}$ and its next elements are the consecutive elements of $V$ up to $\left(t_{1}+1\right)$-th element. Let us denote this element by $v^{2}$. Observe that it belongs to $B$ (obviously, it is different than $v_{1}$ ). Let $V_{2}$ start at $v^{2}$ and let it contain next $\left(t_{2}+1\right)$ elements of the sequence $V$. We denote the last element of $V_{2}$ by $v^{3}$ so $V_{2}=\left(v^{2}, \ldots, v^{3}\right)$. In a similar way we can define the rest of subsequences $V_{3}, \ldots, V_{p}$. The last element of sequence $V_{i}$ we will denote by $v^{i+1}$. It is easy to see that $v^{i} \in B$ for any $i \in\{2, \ldots, p\}$. Thus, a sequence $V_{i}$ contains consecutive elements of $V$, starts at some vertex in $B$ and finishes at another for each $i \in\{1, \ldots, p\}$. Hence, because of the above observation for any $i \in\{1, \ldots, p\}$ the set of vertices of $V_{i}$ describes an open trail $T_{i}$ of length $t_{i}$ in $G$. Moreover, $T_{1}, \ldots, T_{p}$ are edge-disjoint subgraphs of $G$. 
Case II. Let $t_{1}=m_{1} \cdot b, \ldots, t_{l}=m_{l} \cdot b$ for some $l \in\{1, \ldots, p\}$ and for some even integers $m_{1}, \ldots, m_{l}$. Suppose first that $l \geq 2$ and let $m:=m_{1}+\ldots+m_{l}$. Then, consider sequences:

$$
\begin{gathered}
V^{\prime}:=\left(x_{m}, v_{1}, x_{1}, v_{2}, x_{2}, v_{3}, x_{1}, \ldots, x_{1}, v_{b}, x_{2},\right. \\
\left.v_{1}, x_{3}, v_{2}, x_{4}, v_{3}, x_{3}, \ldots, x_{3} v_{b}, x_{4}, \ldots, v_{1}, x_{m-1}, \ldots, x_{m-1}, v_{b}, x_{m}\right)
\end{gathered}
$$

and

$$
\begin{gathered}
V^{\prime \prime}:=\left(v_{1}, x_{m+1}, v_{2}, x_{m+2}, v_{3}, x_{m+1}, \ldots, x_{m+1}, v_{b}, x_{m+2}\right. \\
\left.\ldots, v_{1}, x_{a-1}, \ldots, x_{a-1}, v_{b}, x_{a}, v_{1}\right)
\end{gathered}
$$
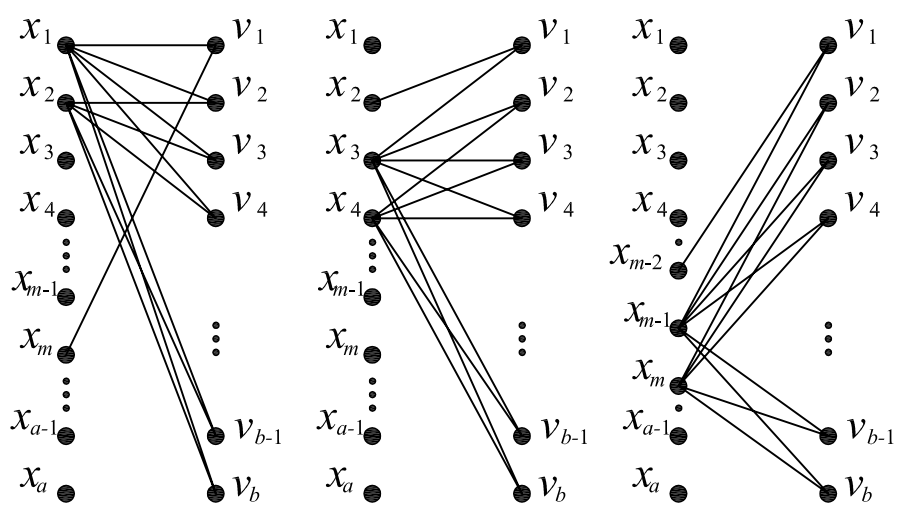

Figure 3: Sequence $V^{\prime}$.
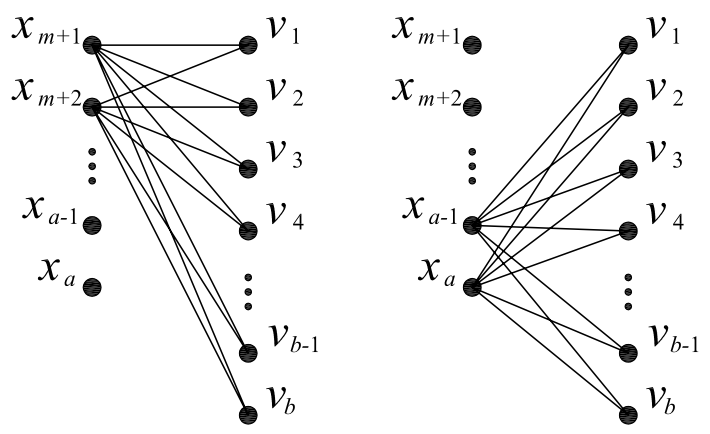

Figure 4: Sequence $V^{\prime \prime}$. 
These sequences of vertices create edge-disjoint closed trails in $G$. Let $G^{\prime}$ be a subgraph of $G$ induced by the set of edges in $V^{\prime}$. Hence $V^{\prime}$ is an Eulerian trail for $G^{\prime}$ which is a complete bipartite subgraph of $G$. Let us part $V^{\prime}$ into disjoint subsequences $V_{1}:=\left(x_{m}, \ldots, x_{m_{1}}\right), V_{i}:=\left(x_{m_{1}+\ldots+m_{i-1}}, \ldots, x_{m_{1}+\ldots+m_{i}}\right)$ for $i \in\{2, \ldots, l-1\}$ and $V_{l}:=\left\{x_{m_{1}+\ldots+m_{l-1}}, \ldots, x_{m}\right)$. The sets of vertices of $V_{i}$ create edge-disjoint open trails $T_{1}, \ldots, T_{l}$ of lengths $t_{1}, \ldots, t_{l}$ (see fig. 3 ).

Let $G^{\prime \prime}$ be a graph described by the sequence of vertices of $V^{\prime \prime}$. Observe that $G^{\prime \prime}$ is also a complete bipartite subgraph of $G$ with two disjoint sets of vertices $C:=A \backslash\left\{x_{1}, \ldots, x_{m}\right\}$ and $B$. The edges of $V^{\prime \prime}$ induce an Eulerian trail for $G^{\prime \prime}$ so we can define the edge-disjoint open trails $T_{l+1}, \ldots, T_{p}$ of lengths $t_{l+1}, \ldots, t_{p}$ in $G^{\prime \prime}$ analogously as in case I (see fig 4 ). Obviously, $T_{1}, . ., T_{p}$ are edge-disjoint open trails in $G$.

Let us assume now that $l=1$. Hence, $t_{1}=m \cdot b$ for some even integer $m$ and $t_{i}$ is not an even multiplicity of $b$ for any $i \in\{2, \ldots, p\}$. Let us consider sequences $V^{\prime \prime \prime}$ (see fig. 5) and $V^{I V}$ (see fig. 6) such that:

$$
V^{\prime \prime \prime}:=\left(v_{1}, x_{1}, v_{2}, x_{2}, v_{3}, x_{1}, v_{4}, x_{2}, v_{5} \ldots, x_{m-1}, v_{b}, x_{a}, v_{b-1}\right)
$$

and

$$
\begin{gathered}
V^{I V}:=\left(v_{b-1}, x_{a-1}, v_{b-2}, x_{a}, v_{b-3}, x_{a-1}, v_{b-4}, x_{a}, \ldots, v_{1}, x_{a-1}, v_{b}\right. \\
x_{a-2}, v_{b-1}, x_{a-3}, v_{b-2}, \ldots, x_{a-2}, v_{1}, x_{a-3}, v_{b}, \ldots \\
\left.x_{m+2}, v_{b-1}, x_{m+1}, v_{b-2}, \ldots, x_{m+2}, v_{1}, x_{m+1}, v_{b}, x_{m}, v_{1}\right) .
\end{gathered}
$$

Observe that the sequence of vertices of $V^{\prime \prime \prime}$ creates an open trail $T_{1}$ of length $t_{1}$. Moreover, with the single exception of $\left\{v_{1}, x_{m+1}, v_{b}, x_{m}\right\}$, every other subsequence which contains consecutive vertices of $V^{I V}$ and start and finish at the same vertex $v_{i}$ for any $i \in\{1, \ldots, b\}$ induces a closed trail of length $k \cdot b$ for some even integer $k$. The only exception is the set of last four vertices $\left\{v_{1}, x_{m+1}, v_{b}, x_{m}\right\}$ which induces a closed trail of length four in $G$. Suppose now that there exists $j \in\{2, \ldots, p\}$ such that $t_{j} \neq 4$. Without loss of generality we can assume that $t_{p} \neq 4$. For such admissible sequence $\tau$, applying analogous methods as in case I, we can define the open trails $T_{2}, \ldots, T_{p}$ of length $t_{2}, \ldots, t_{p}$ in $G$ such that $T_{1}, \ldots, T_{p}$ are edge-disjoint subgraphs of $G$. Assume now that $t_{i}=4$ for any $i \in\{2, \ldots, p\}$ and $b>4$. The edges of a sequence

$$
V^{V}:=\left(v_{2}, x_{2}, v_{3}, x_{1}, v_{4}, x_{2}, v_{5}, \ldots, x_{m-1}, v_{b}, x_{a}, v_{b-1}, x_{a-1}, v_{b-2}\right)
$$



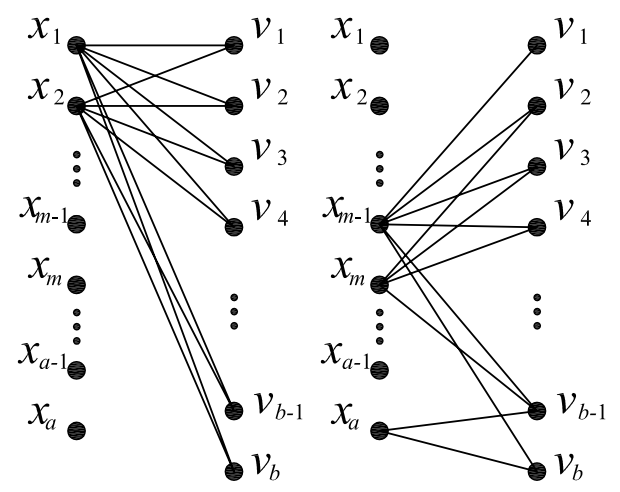

Figure 5: Sequence $V^{I I I}$.
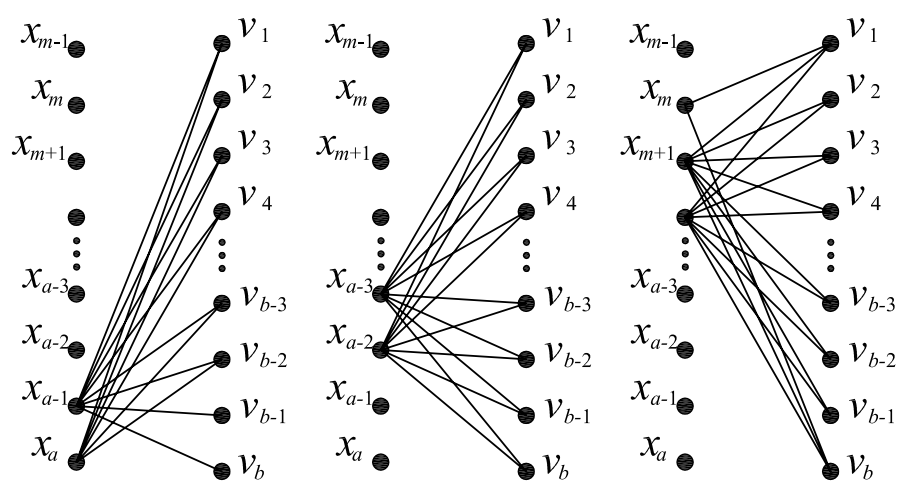

Figure 6: Sequence $V^{I V}$.

induce an open trail of length $t_{1}$ in $G$. Consider a sequence

$$
\begin{gathered}
V^{V I}:=\left(v_{b-2}, x_{a}, v_{b-3}, x_{a-1}, v_{b-4}, x_{a}, \ldots\right. \\
\left.v_{1}, x_{a-1}, v_{b}, x_{a-2}, v_{b-1}, x_{a-3}, v_{b-2}, \ldots, x_{m+1}, v_{b}, x_{m}, v_{1}, x_{1}, v_{2}\right) .
\end{gathered}
$$

(see fig. 7 and 8) Let us part $V^{V I}$ into $(p-1)$ sets, each of them containing five consecutive elements of it. Then these sets induce edge-disjoint open trails of length four in $G$. A decomposition of $G=K_{4,4}$ into edge-disjoint open trails for $\tau=(8,4,4)$ we show in the figure 9 .

B. Suppose now that some of elements of $\tau$ are odd. Without loss of generality we can assume that $t_{1}, \ldots, t_{l}$ are odd for some $l \leq p$ and $t_{l+1}, \ldots, t_{p}$ are even. Observe that $l$ is even so there exists a positive number $k$ such that 

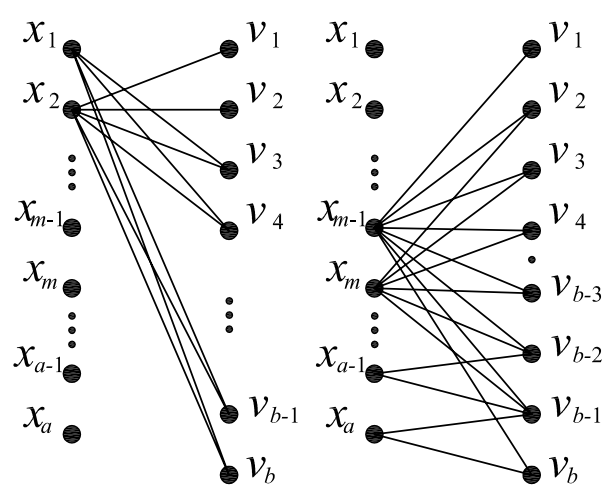

Figure 7: Sequence $V^{V}$.
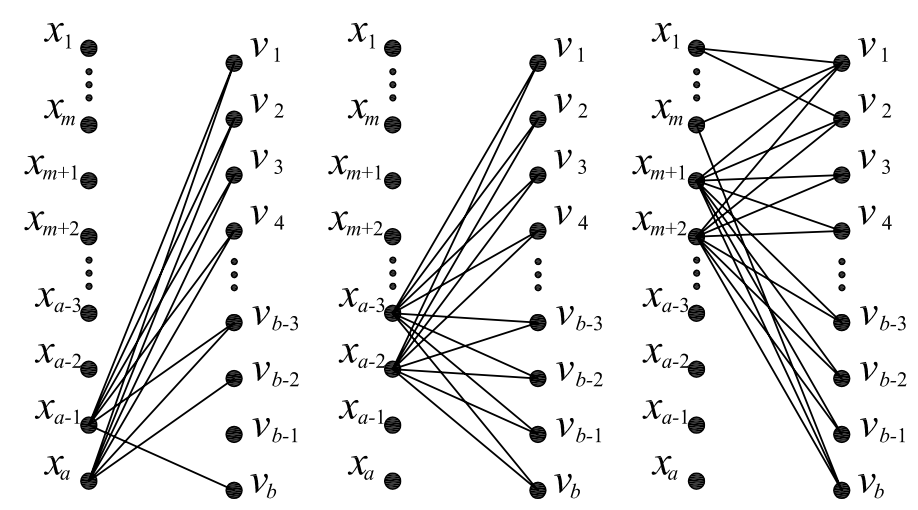

Figure 8: Sequence $V^{V I}$.

$l=2 k$. Let us define $d_{i}:=t_{2 i-1}+t_{2 i}$ for $i \in\{1, \ldots, k\}$. Consider a sequence $\tau^{\prime}:=\left(d_{1}, \ldots, d_{k}, t_{2 k+1}, \ldots, t_{p}\right\}$. Applying the same arguments as above $G$ is decomposable into open trails $D_{1}, \ldots, D_{k}, T_{2 k+1}, \ldots T_{p}$ of lengths $d_{1}, \ldots, d_{k}$, $t_{2 k+1}, \ldots, t_{p}$. It is easy to observe that each open trail $D_{j}$ we can part into two edge-disjoint open trails $T_{2 j-1}, T_{2 j}$ of lenghts $t_{2 j-1}, t_{2 j}$. Hence, $T_{1}, \ldots, T_{p}$ is a $G$-realization of $\tau$ and the proof is finished.

Let us consider now complete bipartite graphs $K_{a, a}$ for an odd $a$. By the previous theorem such graphs are not arbitrarily decomposable into open trails but we can prove the following theorem:

Theorem 5 For any odd a the graph $K_{a, a}^{\prime}$ is decomposable into open trails 


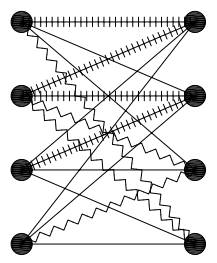

Figure 9: A decomposition of $K_{4,4}$ into edge-disjoint open trails for $\tau=$ $(8,4,4)$.

of lengths $t_{1}, \ldots, t_{p}$ for each admissible sequence $\tau=\left(t_{1}, \ldots, t_{p}\right)$.

Let $G$ be a bipartite graph $K_{a, a}^{\prime}$ with odd $a$. Observe that $p>1$, because there does not exist an open trail of length $\left(a^{2}-a\right)$ in $K_{a, a}^{\prime}$. Let $A:=\left\{x_{1}, \ldots, x_{a}\right\}$ and $B:=\left\{v_{1}, \ldots, v_{a}\right\}$. Let $I_{a}$ be the matching such that $x_{i} v_{j} \in I_{a}$ if and only if $j=i$. Let $\tau=\left(t_{1}, \ldots, t_{p}\right)$ be a sequence of positive integers such that $\sum_{i=1}^{p} t_{i}=a^{2}-a$ and $p \geq 2$. The proof of this theorem is analogous to the proof of Theorem 4 .

Let us suppose first that $t_{i}$ is even for any $i \in\{1, \ldots, p\}$. We consider two cases:

Case I. Assume now that $t_{i}$ is not an even multiplicity of $a$ for any $i \in$ $\{1, \ldots, p\}$. Let us consider a sequence (see fig. 10)

$$
\begin{gathered}
U:=\left(v_{1}, x_{a}, v_{2}, x_{1}, v_{3}, x_{2}, \ldots, v_{1}, v_{a}, x_{2}, v_{1}, x_{3}, v_{2}, x_{4}, v_{3}, x_{a}, v_{4}, \ldots, x_{3}, v_{a}, x_{4}, \ldots,\right. \\
v_{1}, x_{i}, v_{2}, x_{i+1}, \ldots, x_{i+1}, v_{i}, x_{a}, v_{i+1}, x_{i}, v_{i+2}, x_{i+1}, \ldots, x_{i}, v_{a}, x_{i+1}, \ldots, \\
\left.v_{1}, x_{a-2}, v_{2}, x_{a-1}, \ldots, x_{a-1}, v_{a-2}, x_{a}, v_{a-1}, x_{a-2}, v_{a}, x_{a-1}\right) .
\end{gathered}
$$

This sequence of vertices creates an Eulerian trail in $K_{a, a}-I_{a}$. Let $W=$ $\left(w_{1}, \ldots, w_{k}\right) \subset V$ be a subsequence of consecutive elements of $U$ such that $w_{1}=w_{k}=v_{i}$ for some $i \in\{1, \ldots, a\}$. The sequence of vertices in $W$ describes a closed trail in $K_{a, a}-I_{a}$ of length $m \cdot a$ for some even $m \leq a$.

We will define subsequences $V_{1}, \ldots, V_{p}$ of $U$ analogously like in the proof of Theorem 4. So let $V_{1}$ contain $\left(t_{1}+1\right)$ first elements of $U$. Hence it starts at $v_{1}$ and its next elements are the consecutive elements of $U$ up to $\left(t_{1}+1\right)$-th element. Let us denote this element of $B$ by $v^{2}$. Let $V_{2}$ start at $v^{2}$ and let it contain next $\left(t_{2}+1\right)$ elements of sequence $U$ and so on. For each $i \in\{1, \ldots, p\}$ the sequence of vertices of $V_{i}$ creates an open trail $T_{i}$ of length $t_{i}$ in $G$ and $T_{1}, \ldots, T_{p}$ are edge-disjoint subgraphs of $G$. 


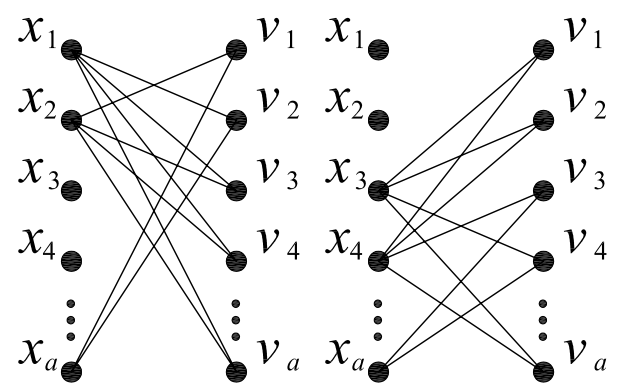

Figure 10: Sequence $U$.

Case II. Let $t_{1}=m_{1} \cdot a, \ldots, t_{l}=m_{l} \cdot a$ for some $l \in\{1, \ldots, p\}$ and for some even integers $m_{1}, \ldots, m_{l}$. Suppose first that $l \geq 2$ and let $m:=m_{1}+\ldots+m_{l}$. Then, consider sequences

$$
\begin{gathered}
U^{\prime}:=\left(x_{m}, v_{1}, x_{a}, v_{2}, x_{2}, v_{3}, x_{1}, \ldots, x_{1}, v_{a}, x_{2}, v_{1}, x_{3}, v_{2}, x_{4}, v_{3}, x_{a}, v_{4}, x_{3}, \ldots,\right. \\
x_{3}, v_{a}, x_{4}, \ldots, v_{1}, x_{i}, v_{2}, x_{i+1}, \ldots, x_{i+1}, v_{i}, x_{a}, v_{i+1}, x_{i}, v_{i+2}, x_{i+1}, \ldots, x_{i}, v_{a}, x_{i+1}, \ldots, \\
\left.v_{1}, x_{m-1}, v_{2}, x_{m}, \ldots, x_{m}, v_{m-1}, x_{a}, v_{m}, x_{m-1}, v_{m+1}, x_{m}, \ldots, x_{m-1}, v_{a}, x_{m}\right)
\end{gathered}
$$

and

$$
\begin{gathered}
U^{\prime \prime}:=\left(v_{1}, x_{m+1}, v_{2}, x_{m+2}, v_{3}, x_{m+1}, \ldots, x_{m+2}, v_{m+1}, x_{a}, v_{m+2}, x_{m+1}, v_{m+3}, x_{m+2}, \ldots,\right. \\
x_{m+1}, v_{a}, x_{m+2}, \ldots, v_{1}, x_{i}, v_{2}, x_{i+1}, \ldots, \\
x_{i+1}, v_{i}, x_{a}, v_{i+1}, x_{i}, v_{i+2}, x_{i+1}, \ldots, x_{i}, v_{a}, x_{i+1}, \ldots, v_{1}, x_{a-2}, v_{2}, x_{a-1}, \ldots, \\
\left.x_{a-1}, v_{a-2}, x_{a}, v_{a-1}, x_{a-2}, v_{a}, x_{a-1}, \ldots, v_{1}, x_{a-1}, \ldots, x_{a-1}, v_{b}, x_{a}, v_{1}\right) .
\end{gathered}
$$

These sequences of vertices create edge-disjoint closed trails in $G$. Let $G^{\prime}$ be a subgraph of $G$ created by the sequence of vertices of $U^{\prime}$. Hence $U^{\prime}$ is an Eulerian trail for $G^{\prime}$. Let us part $U^{\prime}$ into disjoint subsequences $V_{1}:=$ $\left(x_{m}, \ldots, x_{m_{1}}\right), V_{i}:=\left(x_{m_{1}+\ldots+m_{i-1}}, \ldots, x_{m_{1}+\ldots+m_{i}}\right)$ for $i \in\{2, \ldots, l-1\}$ and $V_{l}:=$ $\left\{x_{m_{1}+\ldots+m_{l-1}}, \ldots, x_{m}\right)$. The squences $V_{i}$ create the sets of vertices of edgedisjoint open trails $T_{1}, \ldots, T_{l}$ of lengths $t_{1}, \ldots, t_{l}$ (see fig. 11).

Now, let $G^{\prime \prime}$ be a graph described by the sequence of vertices of $U^{\prime \prime}$. Observe that $G^{\prime \prime}$ is a bipartite subgraph of $G$ with two disjoint sets of vertices $C:=A \backslash\left\{x_{1}, \ldots, x_{m}\right\}$ and $B$ (see fig. 12). The edges of $U^{\prime \prime}$ induce an Eulerian trail for $G^{\prime \prime}$ so we can define the edge-disjoint open trails $T_{l+1}, \ldots, T_{p}$ of lengths 

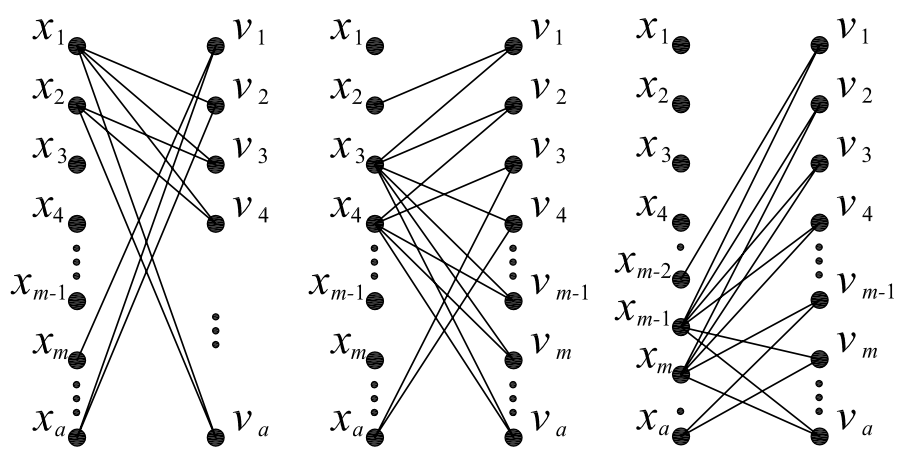

Figure 11: Sequence $U^{\prime}$.

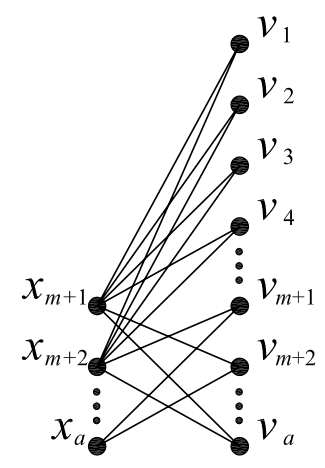

Figure 12: Sequence $U^{\prime \prime}$.

$t_{l+1}, \ldots, t_{p}$ in $G^{\prime \prime}$

Let us assume now that $l=1$. Hence, $t_{1}=m \cdot a$ for some even integer $m$ and $t_{i}$ is not an even multiplicity of $a$ for any $i \in\{2, \ldots, p\}$. Let us consider sequences

$$
U^{\prime \prime \prime}:=\left(v_{1}, x_{a}, v_{2}, x_{2}, v_{3}, x_{1}, v_{4}, x_{2}, v_{5}, \ldots, x_{m-1}, v_{a}, x_{a-2}, v_{a-1}\right)
$$

and

$$
\begin{gathered}
U^{I V}:=\left(v_{a-1}, x_{a}, v_{a-2}, x_{a-1}, v_{b-3}, \ldots, v_{1}, x_{a-1}, v_{a}, \ldots\right. \\
x_{a-4}, v_{a-1}, x_{a-3}, v_{a-2}, x_{a-4}, v_{a-3}, x_{a}, v_{a-4}, x_{a-3}, v_{a-5}, \ldots, \\
\left.x_{m+1}, v_{a-1}, x_{m+2}, v_{a-2}, x_{m+1}, \ldots, v_{1}, x_{m+2}, v_{a}, x_{m}, v_{1}\right) .
\end{gathered}
$$

Observe that the edges of $U^{\prime \prime \prime}$ induce an open trail $T_{1}$ of length $t_{1}$ (see 

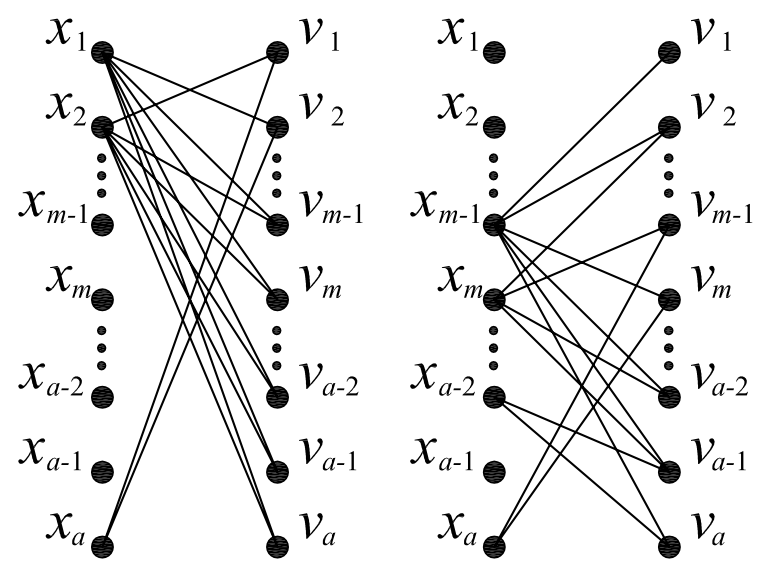

Figure 13: Sequence $U^{\prime \prime \prime}$.

fig. 13). Moreover, with the single exception of $\left\{v_{1}, x_{m+2}, v_{a}, x_{m}\right\}$, every other subsequence which contains consecutive vertices of $U^{I V}$ and start and finish at the same vertex $v_{i}$ for any $i \in\{1, \ldots, b\}$ induces a closed trail of length $k \cdot a$ for some even integer $k$ (see fig. 14). The only exception is the set of last four vertices $\left\{v_{1}, x_{m+2}, v_{a}, x_{m}\right\}$ which induces a closed trail of length four in $G$. Suppose that there exists $j \in\{2, \ldots, p\}$ such that $t_{j} \neq 4$. Without loss of generality we can assume that $t_{p} \neq 4$. For such admissible sequence $\tau$ we can define the open trails $T_{2}, \ldots, T_{p}$ of length $t_{2}, \ldots, t_{p}$ in $G$ such that $T_{1}, \ldots, T_{p}$ are edge-disjoint subgraphs of $G$. Assume now that $t_{i}=4$ for any $i \in\{2, \ldots, p\}$. The vertices in a sequence

$$
U^{V}:=\left(v_{2}, x_{1}, v_{3}, x_{2}, v_{4}, x_{1}, v_{5}, \ldots, x_{m-1}, v_{a}, x_{a-2}, v_{a-1}, x_{a}, v_{a-2}\right)
$$

create an open trail of length $t_{1}$ in $G$ (see fig. 15). Consider a sequence

$$
\begin{gathered}
U^{V I}:=\left(v_{a-2}, x_{a-1}, v_{a-3}, x_{a-2}, v_{a-4}, \ldots,\right. \\
x_{a-2}, v_{1}, x_{a-1}, v_{a}, x_{a-4}, v_{a-1}, x_{a-3}, v_{a-2}, x_{a-4}, v_{a-3}, \ldots, \\
\left.x_{m+1}, v_{1}, x_{m+2}, v_{a}, x_{m}, v_{1}, x_{a}, v_{2}\right) .
\end{gathered}
$$

Let us part $U^{V I}$ into $(p-1)$ sets, each of them containing five consecutive elements of it. Then these sets induce edge-disjoint open trails of length four in $G$ (see fig. 16).

Suppose now that some of elements of $\tau$ are odd. It is obvious that there 


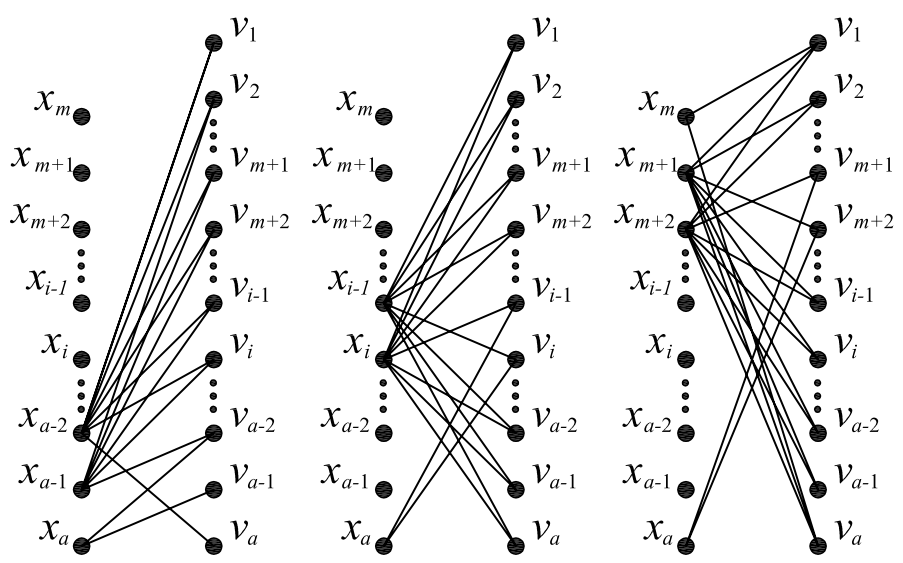

Figure 14: Sequence $U^{I V}$.

is an even number of odd elements in $\tau$. Analogously like in Theorem 4 we can "glue" odd parts creating an element of even length. Hence the proof is finished.

\section{References}

[1] P.N. Balister, Packing Circuits into $K_{n}$, Combin. Probab. Comput. 10 (2001) 463-499.

[2] P.N. Balister and B. Bollobàs, R.H. Schelp, Vertex distinguishing colorings of graphs with $\Delta(G)=2$, Discrete Mathematics 252 (2002) 17-29.

[3] S. Cichacz, Decomposition of complete bipartite digraphs and even complete bipartite multigraphs into closed trails, Discussiones Mathematicae Graph Theory 27 (2) (2007) 241-249.

[4] M. Horňák and M. Woźniak, Decomposition of complete bipartite even graphs into closed trails, Czechoslovak Mathematical Journal 128 (2003) $127-134$. 


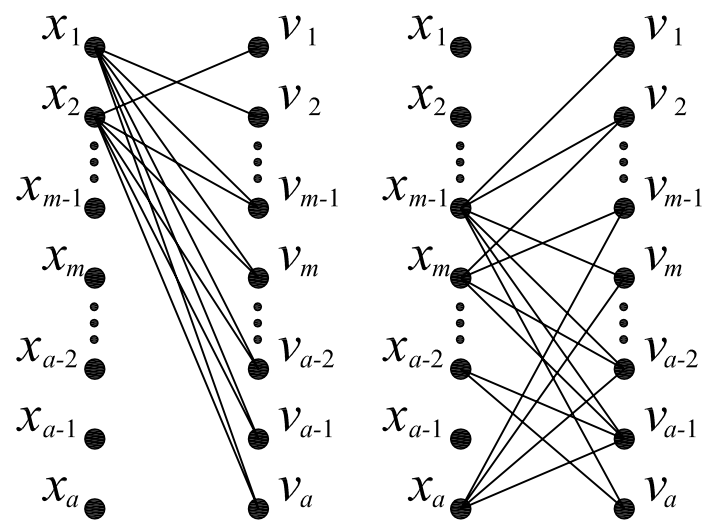

Figure 15: Sequence $U^{V}$.

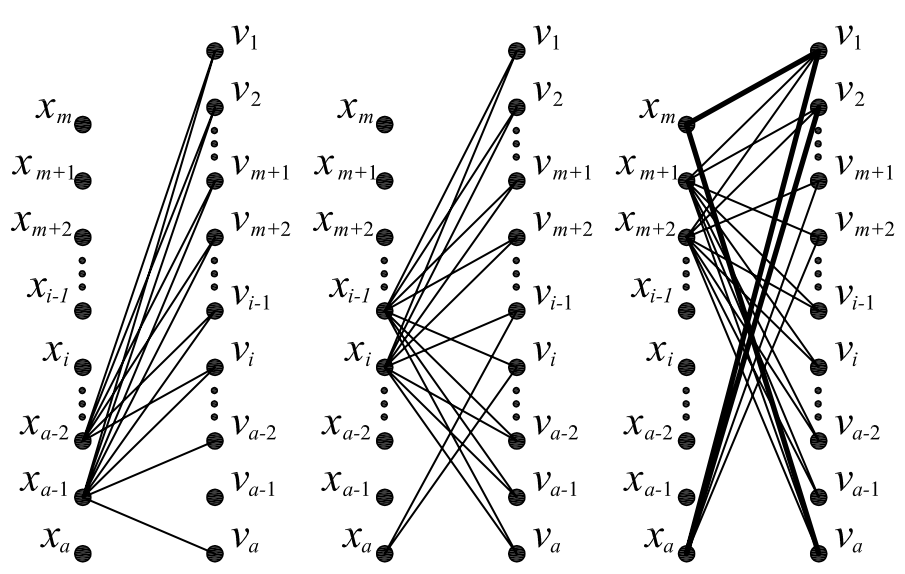

Figure 16: Sequence $U^{V I}$. 